\title{
Advancements in farming and related activities with the help of artificial intelligence: a review
}

\author{
Sidhu, K. S., ${ }^{1} \bowtie$ Gill, A.S., ${ }^{1}$ Arora, A., ${ }^{1}$ Singh, R., ${ }^{2}$ Singh, G., ${ }^{1}$ Verma, M. K., ${ }^{1}$ Kaur, B. ${ }^{3}$
}

Received: 06.11.2020

Revised: 12.01.2021

Accepted: 20.01.2021

\begin{abstract}
The demands of population are gradually increasing rapidly. Most manufacturing and processing industries face obstacles or problems to fulfil this daily increase in demand. Even if the demand can be managed, the transmission of diseases through these sectors is also a considerable concern. Many new technologies are being used to avoid unbearable consequences, which arise because of human-to-human contact or interaction. Higher firms adopt a ton of computer software and other modern technologies to get rid of such problems. Farming, one among the essential professions is also being impacted; however, human beings have come a long way since they started farming. This paper examines how one of the latest technologies, Artificial Intelligence, has helped improve farming, agriculture, and related tasks to increase yield and productivity. How Animal Farming, Detection of Diseases in Plants, Grading processes of Agricultural Foods, and quality check of vegetables and fruits, have advanced with the aid of Artificial Intelligence, is summed up in the subsequent sections.
\end{abstract}

Key Words: Agriculture, artificial intelligence, computer vision, farming, diseases, machine learning

\section{Introduction}

Diseases spreading by viruses, bacteria, fungi, and other micro-organisms are becoming a huge concern and challenge for humankind. Most of the infections spread by physical contact. COVID-19, a viral infection that has become a big challenge for the world has also caused an enormous disturbance in food processing industries where human contact is unavoidable (Sain et al., 2020). This, in turn, is not only impacting financial aspects but also man, material and environmental factors. With Artificial Intelligence's help, one can solve such problems of human contact and uplift humankind's safety factor by complementing manual labour with new-age technology and computer software (Marinoudi et al., 2019; Singh et al., 2020). The factor of hygiene can also be improved by replacing manual labour with the help of machines/equipment using Artificial Techniques and principles in different sectors and industries like production, packaging, printing, etc. As a result, the production or yield will increase as well as the time of processing and

\section{Author's Address}

${ }^{1}$ Department of Computer Science and Engineering

${ }^{2}$ Department of Mechanical Engineering

${ }^{3}$ Department of Agricultural Engineering

Baba Banda Singh Bahadur Engineering, Punjab, India

E-mail.: karamveersidhu14@gmail.com cost of production per piece will reduce. Occupational injuries and human errors are also a big concern in these sectors. Since programming works by using predefined operators and functions and the computer is capable of doing the same work tirelessly, infinitely and more accurately, all these problems are being tackled effectively with Artificial Intelligence techniques which are also playing a profound and scientific role for the upliftment and betterment of the society (Blackwell, 2002).Being one of the most important professions, farming also forms an essential aspect of sustenance for the entire population. The introduction of new technologies and better techniques has changed farming and its yield, improving it substantially. With an increasing population and the agricultural land becoming even scarcer, humans have to be more innovative than before to produce maximum yield. Artificial Intelligence; the intelligence demonstrated or displayed by machines, has solved many problems and has helped make the efficient utilisation of the limited resources available. It has helped to advance and develop numerous industries and fields, including agriculture and farming. Machine Learning is one of the Artificial Intelligence 
applications that equip machines with the capability to learn naturally and improve from experience without the requirement to program them explicitly (Michalski et al., 1983). Below are a few uses of artificial intelligence (AI) in agriculture/farming: -Fruit picking in quicker time with least human resources.

- Crop/yield analysis in an efficient manner in lesser time.

-Identifying and eradicating weeds in an efficient manner, with least wastage and safe manner.

-Soil Sample analysis report will help identify the type of defects in soil and select the type of fertiliser.

-Real-time weather condition data capture and forecast, especially relative humidity, temperature, rain, and solar radiation. When combined with the AI, the data will be able to prevent crop losses and /or increase yield.

-Technology can be used in creating a smart livestock monitoring system and thus help in productivity increase.

\section{Machine learning (ml) in animal farming}

To better understand the well-being of livestock, ML is penetrating farming. Dutta et al. (2015) have presented an ML model that classifies animal behaviour based on collar sensors' data. Many unsupervised and supervised ML models have been discussed, but the most accurate is the bagging ensemble classification with Tree learner. The model's accuracy was $96 \%$. Behaviour like resting, grazing, walking, ruminating were tracked for classification. The study showed that supervised machine learning technology could be inducted for accurate classification of cattle and provides immense potential in gauging animal health problems such as lameness. Pegorini et al. (2015) have presented a system that classifies chewing patterns of grazing animals. Ingestive behaviour in animals that graze is of extreme importance for better growth and improved health. In the study, food intake patterns were considered. The biomechanical strain occurring during jaw movements was measured using data collected with optical fibre Bragg grating sensors (FBG). The developed model's classification accuracy is $94 \%$. Matthews et al. (2017) have discussed a monitoring system which is automated using ML. The system tracks the pig movements using depth video cameras. The cameras monitor activities like standing, moving, drinking and feeding. This method has the potential of changing how livestock breeders look after their livestock. Craninx et al. (2008) has described a machine learning technique that predicts the rumen fermentation pattern from milk fatty acids. Morales et al. (2016) used Support Vector Machines (SVM) to detect problems early in commercial eggs production. The technique achieved an accuracy of 0.9854 . The egg production data from a farm were utilised for the study. Alonso et al. (2015) also show the use of SVM to foresee the weight of livestock animals. Bovines of different ages and breeds were used to collect data. Livestock breeders should have the tools to foresee the weight of their livestock accurately. A model that predicts the weight of carcass is presented by Alonso et al. (2013). The study was done on the beef breed.

The carcass weights can be predicted 4-5 months before the slaughter day using the developed model. The authors used SVM for regression for developing the model. Hansen et al. (2018) aim to tackle the problems with the current method of radio frequency identification (RIFD) tags for identification of animals, which is time-consuming and uncomfortable for the animals. A convolution neural network $(\mathrm{CNN})$ model was trained using digital images for pig face recognition.

\section{Techniques using artificial intelligence to detect plant diseases}

DCNN (Deep convolutional neural networks), also called ConvNet, belongs to the deep neural network category, which is generally used as a visual imagery technique. It is currently most prevalent in detecting plant disease as it helps in tracking plantrelated pest diseases with great efficiency. The technique is more preferable as it's not labour intensive so it could be a practical approach for computational analysis of plant disease. Recently, a study reported Artificial Intelligence-based DCNN and transfer learning system to provide accurate predictions for diseases and pest detection in banana (Selvaraj et al., 2019). Convolutional neural network model named LeNet detects and identify diseases in tomato leaves ( $\mathrm{T} \mathrm{m}$ et al., 2018). Apple leaf diseases were identified based on Deep 
Convolutional Neural Networks using AlexNet architecture (Liu et al., 2018). 3D CNN model is used for charcoal rot disease as it can automatically learn Spatio-temporal features without handcrafting, with high accuracy (Nagasubramanian et al., 2019). Hyperspectral imaging is a preferable technique to identify various crop diseases as it is non-invasive and a fast detection method. Under this push, broom technology and snapshot technology are the recent ones helping in the proper analysis of crop condition. Hyperspectral imaging as well as machine learning techniques both help to differentiate between healthy capsicum plants and those infected by Tomato Spotted Wilt Virus (TSWV) (Moghadam et al., 2017) and also help to detect the Tobacco Mosaic Virus (TMV) disease in very less time (Zhu et al., 2017). Three Dimensional Hyperspectral plant models had been studied to detect Cercospora leaf spot disease in sugar beet leaves (Roscher et al., 2016). Hyperspectral reflection and transmission measurements were conducted to study Blumeria graminis $\mathrm{f}$. sp hordei infection in barley (Thomas et al., 2017). Artificial Intelligence-based thermal and stereo image technology supports the classification of powdery mildew disease of tomato (Prince et al., 2015). Mokhtar et al. (2015) reported a tomato yellow leaf curl virus classification using supervised machine learning support vector machine models pipeline (Mokhtar et al., 2015). The problem of pest diseases can be tackled with an innovative app launched, named Plantix app (Plant doctor) in which the photo of the infected plant is captured, analysed, compared with prior information and then at last treatment options as well as preventive measures are provided to maintain the health of the plant (Tibbets and John., 2018). Similarly, the newest Artificial Intelligence based algorithms for Cloud-based image processing has allowed real-time diagnosis and help the experts to analyse the extent of disease with geographical visualisation (Singh, 2018). The innovation of artificial intelligence nose (electronic nose) is considered a fast and non-invasive technique for detecting plant diseases (Cui et al., 2018). To assess and monitor plant stresses such as pests, diseases, drought, and weeds, unmanned aerial vehicles (UAVs) and drones, are used (Barbedo et al., 2019). UAVs and deep CNN are used to capture high-resolution images of infected plants so that the plant-associated diseases such as a foliar disease in maize caused by Setosphaeria turcica, in the case of Northern leaf blight (NLB) (Wu et al., 2019) and soybean leaf diseases (Tetila et al., 2019) can be easily detected. Spatial and spectral data have been developed on hyperspectral UAV images, using a deep learning model called the multiple inception-racenet models to detect yellow corrosion in wheat (Saleem et al., 2019). Table 1 shows a comparison of various deep learning approaches in terms of different performance metrics. Five kinds of diseases in apple plant were classified and detected with the help of a state-of-the-art CNN model, i.e. VGGinception architecture was successful in detecting apple diseases and also outclasses the activity of other DL architectures like AlexNet, GoogLeNet, several versions of ResNet, and VGG for clarity of diseases in the plants (Jiang et al., 2019). Modern deep learning model helped detect pest infecting rice plants using novel Artificial Intelligence and IoT (Internet of things) methods (Win et al., 2018). Artificial Neural network and diverse image processing techniques are used for the detection of cotton leaf diseases (Ranjan et al., 2015). A Robust Deep-Learning-based detector has been developed to monitor diseases and recognise pests in Tomato Plants' real-time (Fuentes et al., 2017). CNN's Transfer learning is used for identifying plant leaf's diseases using VGGNet already pre-trained on the ImageNet database and Inception module (Chen et al., 2020). This approach is used to recognise the image of cassava disease infected plants and is a fast and affordable strategy for detecting diseases in plants digitally (Ramcharan et al., 2017). To detect diseases subsisting in leaves automatically, Neural Networks are being used. This approach provides accurate detection in leaf and is an important method, in the case of the stem, and root diseases with fewer computation efforts and is also moreover, less labour intensive.

\section{Grading process for agricultural foods using artificial intelligence}

In the present era of advanced technologies, both the hardware and software features reduce human efforts and yield better results. The use of AI in the agriculture sector has become a trend among 
various developed countries. In today's industrial world, the consumer demands products of good quality. The machine vision technique of grading system has been developed to conquer these problems and lessen labour requirements. For a machine vision system, the hardware, as well as the software, is required.

Table 1. Comparison of several Deep Learning approaches in terms of various $p$ erformance metrics (retrieved from Saleem et al., 2019)

\begin{tabular}{|c|c|c|c|}
\hline DL Architectures/Algorithms & Datasets & Selected Plant/s & $\begin{array}{l}\text { Performance Metrics (and } \\
\text { Their Results) }\end{array}$ \\
\hline CNN & PlantVillage & Maize & CA $(92.85 \%)$ \\
\hline AlexNet, GoogLeNet, ResNet & PlantVillage & Tomato & $\begin{array}{l}\text { CA by ResNet which gave } \\
\text { the best value }(97.28 \%)\end{array}$ \\
\hline LeNet & PlantVillage & Banana & CA (98.61\%), F1 (98.64\%) \\
\hline $\begin{array}{l}\text { AlexNet, ALexNetOWTBn, } \\
\text { GoogLeNet, Overfeat, VGG }\end{array}$ & $\mid \begin{array}{l}\text { PlantVillage and } \\
\text { in-field images }\end{array}$ & $\begin{array}{l}\text { Apple, blueberry, banana, } \\
\text { cabbage, cassava, cantaloupe, } \\
\text { celery, cherry, cucumber, corn, } \\
\text { eggplant, gourd, grape, orange, } \\
\text { onion }\end{array}$ & $\begin{array}{l}\text { Success rate of VGG } \\
(99.53 \%) \text { which is the best } \\
\text { among all }\end{array}$ \\
\hline $\begin{array}{l}\text { AlexNet, VGG16, VGG 19, } \\
\text { SqueezeNet, GoogLeNet, } \\
\text { Inceptionv3, InceptionResNetv2, } \\
\text { ResNet50, Resnet101 }\end{array}$ & Real field dataset & Apricot, Walnut, Peach, Cherry & $\begin{array}{l}\text { F1(97.14), Accuracy }(97.86 \\
\pm 1.56) \text { of ResNet }\end{array}$ \\
\hline Inceptionv3 & $\begin{array}{l}\text { Experimental } \\
\text { field dataset }\end{array}$ & Cassava & CA $(93 \%)$ \\
\hline $\mathrm{CNN}$ & \begin{tabular}{|l|} 
Images taken \\
from the research \\
center
\end{tabular} & Cucumber & CA $(82.3 \%)$ \\
\hline $\begin{array}{l}\text { Super-Resolution Convolutional } \\
\text { Neural Network (SCRNN) }\end{array}$ & 1 PlantVillage & Tomato & Accuracy $(\sim 90 \%)$ \\
\hline CaffeNet & $\begin{array}{l}\text { Downloaded } \\
\text { from the internet }\end{array}$ & $\begin{array}{l}\text { Pear, cherry, peach, apple, } \\
\text { grapevine }\end{array}$ & Precision (96.3\%) \\
\hline AlexNet and GoogLeNet & PlantVillage & $\begin{array}{l}\text { Apple, blueberry, bell pepper, } \\
\text { cherry, corn, peach, grape, } \\
\text { raspberry, potato, squash, } \\
\text { soybean, strawberry, tomato }\end{array}$ & $\begin{array}{ll}\text { CA } & (99.35 \%) \\
\text { GoogLeNet }\end{array}$ \\
\hline $\begin{array}{l}\text { AlexNet, GoogLeNet, VGG- 16, } \\
\text { ResNet-50,101, ResNetXt-101, } \\
\text { Faster RCNN, SSD, R-FCN, } \\
\text { ZFNet }\end{array}$ & $\begin{array}{l}\text { Image taken } \\
\text { real fields }\end{array}$ & Tomato & $\begin{array}{l}\text { Precision } \quad(85.98 \%) \text { of } \\
\text { ResNet-50 } \quad \text { with Region } \\
\text { based Fully } \\
\text { Network(R-FCN) }\end{array}$ \\
\hline $\mathrm{CNN}$ & $\begin{array}{l}\text { Bisque platform } \\
\text { of Cy Verse }\end{array}$ & Maize & Accuracy $(96.7 \%)$ \\
\hline DCNN & \begin{tabular}{|lr} 
Images & were \\
taken in real field
\end{tabular} & Rice & Accuracy $(95.48 \%)$ \\
\hline AlexNet, GoogLeNet & PlantVillage & Tomato & $\begin{array}{l}\text { Accuracy }(0.9918 \pm 0.169) \\
\text { of GoogLeNet }\end{array}$ \\
\hline
\end{tabular}

To capture the images of products, hardware like and budding technique, even used for consumers the camera and computer is required. Then features feedback (Tsoumakas, 2018). When a consumer of the respective images are collected through a seeks some changes in a product, he can provide computer, and each and every feature is analysed, feedback, and the machines can automatically yield using Image processing techniques (Elakkiya et al., those desired results. Therefore, the product's 2018). Artificial Intelligence has become prominent quality is easily determined, and it also leads to a 
reduction in time. Also, the new process of "Grading System" seems to be as of good interest for farmers. The Grading system helps to draw outcomes from various surveys and instruct machines according to it. It also helps the producer to track the status of the product until it gets to the customer. All this reduces human efforts and saves precious time. Many users have confirmed that AI has provided better results with errors approaching zero. Artificial Intelligence techniques have also been used to improve the grading system, to get a precise value. The grading system's AI techniques are used to well develop the food industry's industrial products, for instance, the agricultural products. However, the Grading system varies for each product or item as required by either owner or customer(Nychas et al., 2016). The other form of survey is used for segregation of the good quality products from the bad quality products. This feature has emerged out as a boon for the agricultural society. Nowadays, the researchers are working to advance the existing technology by minimising the error in this technique's output. A comparative analysis of fruit grading is shown in Table 2.

Table 2. Comparative analysis of fruit grading (retrieved from Elakkiya et al., 2018)

\begin{tabular}{|l|l|l|l|}
\hline Fruits & Features & Technique & Accuracy \\
\hline Mango & Colour of the image changes. & IR vision sensor and Gaussian Mixture Model. & Not specified \\
\hline $\begin{array}{l}\text { Harumani } \\
\text { Mangoes }\end{array}$ & Shape, colour, weight of the mangoes. & Fourier Based separation model. & $90 \%$ \\
\hline Cashew & $\begin{array}{l}\text { Colour, texture, size and shape of } \\
\text { cashews. }\end{array}$ & $\begin{array}{l}\text { Multi resolutional Wavelet transform and AI } \\
\text { (classifier) of SVM and BPNN }\end{array}$ & $95 \%$ \\
\hline $\begin{array}{l}\text { Cherry } \\
\text { Tomato }\end{array}$ & $\begin{array}{l}\text { Colour, texture, shape (external and } \\
\text { internal) characteristics }\end{array}$ & AI technique of SVM and KNN classifier. & Not specified \\
\hline Peanut & $\begin{array}{l}\text { Shape, texture and colour of peanuts. } \\
\text { Apple }\end{array}$ & $\begin{array}{l}\text { Skin or surface of the apple and colour. } \\
\text { AI technique of FNN and SVM. }\end{array}$ & Not specified \\
\hline
\end{tabular}

Artificial intelligence to check the quality of vegetables and fruits are transported in containers vegetables and fruits

to a special sensor with the help of a conveyor belt.

Automatic identification of vegetables and fruits is achieved with computer vision along with machine learning. Block diagram attributed to the classification process is manifested in figure 1 (Jana et al., 2017). Automatic harvesting is a growing field that collects useful information about growing, cultivating, checking the ripeness of vegetables and fruits, along with other aspects of farming with the help of computer vision and machine intelligence. Fruit picking and sorting are also taking place these days with the help of computer vision. In most farm industries, ripeness is still checked manually by humans but assessing the ripeness level precisely is different even for expert inspectors. In one of Amazon's tests, the evaluators reached dissimilar results in twenty per cent of the tests, despite examining the quality of the same vegetables and fruits. In the automatic ripeness detection system, The sensor appears like a typical camera, but it can gather the imperceptible information to the human eye. Using machine learning's principles, the machine learns about good and bad products with the help of the input provided. New product versions are provided as daily input. Images of the products are captured and then given as data to the machine. It allows the computer to understand the quality of vegetables and fruits.Fruits are split up into different classes: "OK", "Damaged", "Severely Damaged" and "Expired". Cases containing vegetables and fruits of different ripening categories are introduced regularly into the machine. Similarly, employees know only through the screen of how the entire container will be filled. This helps prevent every third case being packed with "damaged" fruits and vegetables or every fourth case with "healthy" fruits. Instead of 
permitting the machine to grasp the sequence without proper product testing, it actually prevents the machine from grasping a pattern.

\section{Figure 1. Block diagram of a classification process} (retrieved from Jana et al., 2017)

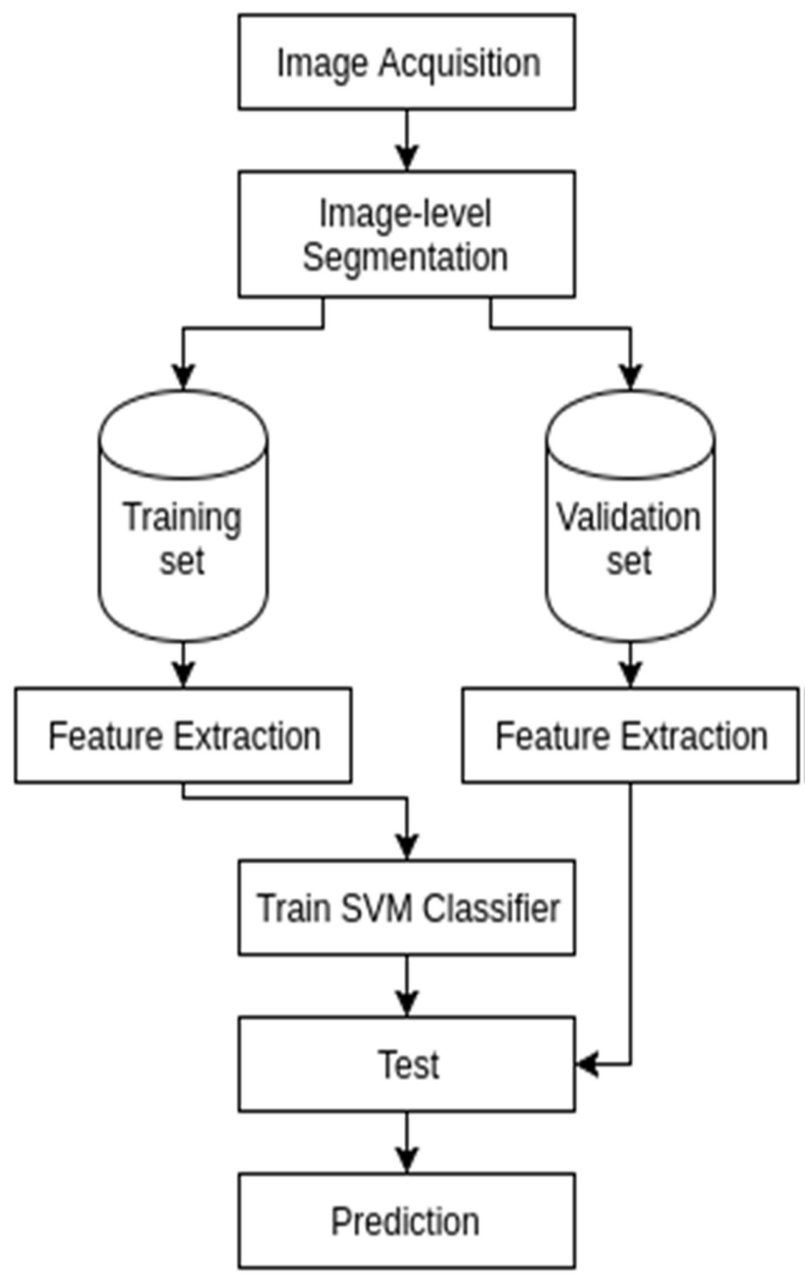

\section{Conclusion}

Artificial Intelligence, one among the modern technologies and a broad field, has a ton of applications. It is extensively used in farming, agriculture and related activities, and aims to achieve more effective and efficient results that otherwise were not possible by following the traditional practices. It is evolving day by day, and scientists are trying to find even better solutions to solve the modern-day problems more optimally and sustainable development.

\section{References}

Barbedo, J.G.A., 2019. A review on the use of unmanned aerial vehicles and imaging sensors for monitoring and assessing plant stresses. Drones, 3(2), p.40. https://doi.org/10.3390/drones3020040

Blackwell, A. F., 2002. What is Programming? University of Cambridge Computer Laboratory. https://ogur.org/1590284993.pdf

Chen, J., Chen, J., Zhang, D., Sun, Y. and Nanehkaran, Y.A., 2020. Using deep transfer learning for image-based plant disease identification. Computers and Electronics in Agriculture, 173, p.105393. https://doi.org/10.1016/j.compag.2020.105393

Cui, S., Ling, P., Zhu, H. and Keener, H.M., 2018. Plant pest detection using an artificial nose system: a review. Sensors, 18(2), p.378. https://doi.org/10.3390/s18020378

Fuentes, A., Yoon, S., Kim, S.C. and Park, D.S., 2017. A robust deep-learning-based detector for real-time tomato plant diseases and pests recognition. Sensors, 17(9), p.2022. https://doi.org/10.3390/s17092022

George-John E Nychas, Efstathios Z Panagou, Fady Mohareb., 2016. Novel approaches for food safety management and communication. Current Opinion in Food Science. Volume 12, December 2016, Pages 13-20 https://www.sciencedirect.com/science/article/pii/S221479 $\underline{9316300777}$

Grigorios Tsoumakas., 2018. Survey of machine learning techniques for food sales prediction. Artif Intell $\operatorname{Rev~52,~441-447.~} \quad$ https://doi.org/10.1007/s10462-018$\underline{9637-z}$

Iván Ramírez Morales, Daniel Rivero Cebrián, Enrique Fernández Blanco, Alejandro Pazos Sierra., 2016. Early warning in egg production curves from commercial hens: A SVM Approach. Computers and Electronics in Agriculture Volume 121, Pages 169-179. https://www.sciencedirect.com/science/article/pii/S0168 $\underline{169915003919}$

Jaime Alonso, Alfonso Villa, Antonio Bahamonde., 2015. Improved estimation of bovine weight trajectories using Support Vector Machine Classification. Computers and Electronics in Agriculture. Volume 110, Pages 36-41. https://www.sciencedirect.com/science/article/pii/S016816 9914002488

Jaime Alonso, Angel Rodriguez Castanon, Antonio Bahamonde., 2013. Support Vector Regression to predict carcass weight in beef cattle in advance of the slaughter. Computers and Electronics in Agriculture. Volume 91, Pages 116-120.

https://www.sciencedirect.com/science/article/pii/S016816991200 $\underline{2232}$ 
Jiang, P., Chen, Y., Liu, B., He, D. and Liang, C., 2019. Realtime detection of apple leaf diseases using deep learning approach based on improved convolutional neural networks. IEEE Access, 7, $\underline{10.1109 / A C C E S S .2019 .2914929}$

Liu, B., Zhang, Y., He, D. and Li, Y., 2018. Identification of apple leaf diseases based on deep convolutional neural networks. Symmetry, 10(1), p.11. https://doi.org/10.3390/sym10010011

Marinoudi, V., Sørensen, C. G., Pearson, S., \& Bochtis, D., 2019. Robotics and labour in agriculture. A context consideration. Biosystems Engineering, 184, 111121. doi:10.1016/j.biosystemseng.2019.06.013

Mark F. Hansena, Melvyn L. Smitha, Lyndon N. Smitha, Michael G. Salterb, Emma M. Baxterc, Marianne Farishc, Bruce Grieved., 2018. Towards on-farm pig face recognition using convolutional neural networks. Computers in Industry. Volume 98, June 2018, Pages 145152.

https://www.sciencedirect.com/science/article/pii/S0166 361517304992

M. Craninxa, V. Fieveza, B. Vlaemincka, B. De Baetsb., 2008. Artificial neural network models of the rumen fermentation pattern in dairy cattle. Computers and Electronics in Agriculture 60(2):226-238.

https://www.researchgate.net/publication/250718986 Artif icial neural network models of the rumen fermentation pattern in dairy cattle

Michalski, R. S., $\quad$ Carbonell, J. G., \& Mitchell, T. M., 1983. Machine Learning published by Springer. https://link.springer.com/content/pdf/10.1007/978-3-66212405-5.pdf

Moghadam, P., Ward, D., Goan, E., Jayawardena, S., Sikka, P. and Hernandez, E., 2017, November. Plant disease detection using hyperspectral imaging. In 2017 International Conference on Digital Image Computing: Techniques and Applications (DICTA) (pp. 1-8). IEEE. https://ieeexplore.ieee.org/abstract/document/82274 $\underline{76}$

Mokhtar, U., Ali, M.A., Hassanien, A.E. and Hefny, H., 2015. Identifying two of tomatoes leaf viruses using support vector machine. In Information Systems Design and Intelligent Applications (pp. 771-782). Springer, New Delhi. DOI: 10.1007/978-81-322-2250-7 77

Nagasubramanian, K., Jones, S., Singh, A.K., Sarkar, S., Singh, A. and Ganapathysubramanian, B., 2019. Plant disease identification using explainable 3D deep learning on hyperspectral images. Plant methods, 15(1), p.98. https://plantmethods.biomedcentral.com/articles/10.1186/s 13007-019-0479-8

https://plantmethods.biomedcentral.com/articles/10.1186/s 13007-019-0475-z
N.Elakkiya, Dr.S.Karthikeyan, T.Ravi., 2018. Survey of grading process for agricultural foods by using Artificial Intelligence https://ieeexplore.ieee.org/abstract/document/8474663/

Pilli, S.K., Nallathambi, B., George, S.J. and Diwanji, V., 2015, February. eAGROBOT-A robot for early crop disease detection using image processing. In 2015 2nd International Conference on Electronics and Communication Systems (ICECS) (pp. 1684-1689). IEEE. https://doi.org/10.1109/ECS.2014.7090754

Prince, G., Clarkson, J.P. and Rajpoot, N.M., 2015. Automatic detection of diseased tomato plants using thermal and stereo visible light images. PloS one, 10(4), p.e0123262. https://doi.org/10.1371/journal.pone.0123262

Ramcharan, A., Baranowski, K., McCloskey, P., Ahmed, B., Legg, J. and Hughes, D.P., 2017. Deep learning for imagebased cassava disease detection. Frontiers in plant science, 8, p.1852. https://doi.org/10.3389/fpls.2017.0185

Ranjan, M., Weginwar, M.R., Joshi, N. and Ingole, A.B., 2015. Detection and classification of leaf disease using artificial neural network. International Journal of Technical Research and Applications, 3(3), pp.331-333. https://www.ijtra.com

Ritaban Dutta, Daniel Smith, Richard Rawnsley, Greg BishopHurley, James Hills, Greg Timms , Dave Henry. 2015. Dynamic cattle behavioural classification using supervised ensemble Classifiers. Computers and Electronics in Agriculture. Volume 111, Pages 18-28. https://www.sciencedirect.com/science/article/pii/S0168 $\underline{169914003123}$

Roscher, R., Behmann, J., Mahlein, A.K., Dupuis, J., Kuhlmann, H. and Plümer, L., 2016. Detection of disease symptoms on hyperspectral 3d plant models. ISPRS Annals of Photogrammetry, Remote Sensing \& Spatial Information Sciences, 3(7). https://doi.org/10.5194/isprsannals-III-7-89-2016

Sain, M., \& Singh, R., Kaur, A. (2020). Robotic Automation in Dairy and Meat Processing Sector for Hygienic Processing and Enhanced Production. Journal of Community Mobilization and Sustainable Development, Vol. 15(3), 543-550, September-December, 2020

Saleem, M.H., Potgieter, J. and Arif, K.M., 2019. Plant disease detection and classification by deep learning. Plants, 8(11), p.468. https://doi.org/10.3390/plants8110468

Selvaraj, M.G., Vergara, A., Ruiz, H., Safari, N., Elayabalan, S., Ocimati, W. and Blomme, G., 2019. AI-powered banana diseases and pest detection. Plant Methods, 15(1), p.92.

Singh, R., Sain, M., Singh, B., Nagi, H. S., \& Bala, N. (2020). Development of a Cost Effective Beverage and FoodServing Robot for Hygienically Outcomes and Human 
Comfort. International Journal of Curent Microbiology and Applied Science 9(5), 247-257.

Stephen G. Matthews, Amy L. Miller, Thomas PlÖtz and Ilias Kyriazakis., 2017. Automated tracking to measure behavioural changes in pigs for health and welfare monitoring. Sci Rep 7, 17582.

https://doi.org/10.1038/s41598-017-17451-6.

Tetila, E.C., Machado, B.B., Menezes, G.K., Oliveira, A.D.S., Alvarez, M., Amorim, W.P., Belete, N.A.D.S., Da Silva, G.G. and Pistori, H., 2019. Automatic recognition of soybean leaf diseases using UAV images and deep convolutional neural networks. IEEE Geoscience and Remote Sensing Letters, 17(5), pp.903-907. https://doi.org/10.1109/LGRS.2019.2932385

Thomas, S., Wahabzada, M., Kuska, M.T., Rascher, U. and Mahlein, A.K., 2017. Observation of plant-pathogen interaction by simultaneous hyperspectral imaging reflection and transmission measurements. Functional Plant Biology, 44(1), pp.2334. https://doi.org/10.1071/FP16127

Tibbets, John H., 2018. From identifying plant pests to picking fruit, AI is reinventing how farmers produce your food. $\quad$ https://www.eco-business.com/news/fromidentifying-plant-pests-to-picking-fruit-ai-is-reinventinghow-farmers-produce-your-food

Tm, P., Pranathi, A., SaiAshritha, K., Chittaragi, N.B. and Koolagudi, S.G., 2018. Tomato leaf disease detection using convolutional neural networks. In 2018 Eleventh International Conference on Contemporary Computing (IC3) (pp. 1-5). IEEE. https://doi.org/10.1109/IC3.2018.8530532

Vinicius Pegorini, Leandro Zen Karam, Christiano Santos Rocha Pitta, Rafael Cardoso, Jean Carlos Cardozo da Silva, Hypolito José Kalinowski, Richardson Ribeiro, Fábio Luiz Bertotti and Tangriani Simioni Assmann., 2015. In Vivo Pattern Classification of Ingestive Behavior in Ruminants Using FBG Sensors and Machine Learning. https://www.ncbi.nlm.nih.gov/pmc/articles/PMC4701289

Win, T.T., 2018. AI and IoT methods for plant disease detection in Myanmar. Doctoral dissertation, Kobe Institute OF Computing. Master thesis, submitted to Markon lab department of information technology.

Wu, H., Wiesner-Hanks, T., Stewart, E.L., DeChant, C., Kaczmar, N., Gore, M.A., Nelson, R.J. and Lipson, H., 2019. Autonomous detection of plant disease symptoms directly from aerial imagery. The Plant Phenome Journal, 2(1), pp.1-9.

Zhu, H., Cen, H., Zhang, C. and He, Y., 2016. Early detection and classification of tobacco leaves inoculated with tobacco mosaic virus based on hyperspectral imaging technique. In 2016 ASABE Annual International Meeting (p. 1). American Society of Agricultural and Biological Engineers. https://doi.org/10.13031/aim.20162460422. 